\title{
Silicon Carbide as a tritium permeation barrier in tungsten plasma-facing components
}

G.M. Wright ${ }^{1 *}$, M.G. Durrett ${ }^{2}$, K.W. Hoover ${ }^{2}$, L.A. Kelser ${ }^{1}$, and D.G. Whyte ${ }^{1}$

${ }^{1}$ MIT Plasma Science and Fusion Center, 77 Massachusetts Ave, Cambridge, MA, 02139, USA

${ }^{2}$ Nanohmics Inc. 6201 E Oltorf St, Austin, TX, 78741, USA

*Corresponding author email: wright@psfc.mit.edu

Corresponding author telephone: (617) 253-5956

Corresponding author address: MIT PSFC, NW17-121, 77 Massachusetts Ave.

Cambridge, MA, USA, 02139

$<<$ Manuscript is submitted as a Letter to the Editor, no abstract is provided $>>$

\section{Introduction}

The control of tritium inventory is of great importance in future fusion reactors, not only from a safety standpoint but also to maximize a reactor's efficiency. Due to the high mobility of hydrogenic species in tungsten (W) one concern is the loss of tritium from the system via permeation through the tungsten plasma-facing components (PFC). This can lead to loss of tritium through the cooling channels of the wall thereby mandating tritum monitoring and recovery methods for the cooling 
system of the first wall. The permeated tritium is then out of the fuel cycle and cannot contribute to energy production until it is recovered and recycled into the system.

Most work on tritium permeation barriers has been focused at the breeding blanket where tritium is bred to maintain a self-sustaining tritium fuel cycle [1-3]. Tritium inventory control is critical there as well to maintain a viable tritium breeding ratio, but there is also a great benefit to reducing permeation of tritium through the PFC (e.g. the first wall). This stems from the fact that the rate at which the tritium recycles between the plasma and solute state in the plasma-facing materials is much larger than any other tritium "loop" in the reactor. This work demonstrates that a thin layer of silicon carbide (SiC) can be used in a W PFC to greatly reduce hydrogenic permeation while maintaining structural integrity of the PFC through rapid thermal anneal and thermal cycling tests up to $1023 \mathrm{~K}$.

2. Experiment

\subsection{Target fabrication}

PFC in a fusion reactor are tasked with simply surviving the particle and heat exhaust from a tokamak plasma. Ideally, they will not experience macroscopic erosion such as de-lamination and their sputtering rate must allow them to survive for an acceptable component life-time. They must also handle very high heat loads up to $\sim 20 \mathrm{MW} / \mathrm{m}^{2}$ and plasma fluxes up to $10^{24} \mathrm{ions} / \mathrm{m}^{2} \mathrm{~s}$. This means that any permeation barrier to be used in a W PFC must have high thermal conductivity so the heat handling abilities are not compromised, it must be electrically conductive to prevent charging from the plasma which could lead to harmful arcing and it must 
have a coefficient of thermal expansion close to $\mathrm{W}$ to avoid any de-lamination or structural issues from thermal cycling, which could compromise the life-time of the PFC. While none of the permeation barriers proposed for the blanket modules fit all these criteria, $\mathrm{SiC}$ can match all these properties for $\mathrm{W}$ very well [4-9] while also having a hydrogenic diffusivity many orders of magnitude times lower than $\mathrm{W}$ at 550 K [10-12], see Table 1.

The thermal conductivity and hydrogenic diffusivity of $\mathrm{SiC}$ is sensitive to crystallinity, phase, morphology, and impurity content, which leads to the range of values given in table 1 . The scatter in thermal conductivities is will likely be insignificant towards this work as the SiC permeation barrier will be a thin layer in a bulk sample and have negligible impact on the overall thermal conductivity of the PFC in its entirety. The wide range of diffusivity values can be a more important since diffusivity is related to permeation directly through the solubility of the material. Table 1 shows that the hydrogenic diffusivity is commonly much lower in SiC than in W but the exact difference can vary by orders of magnitude, which has to do with various $\mathrm{SiC}$ crystallinities and fabrication methods. It can be noted as well that measured diffusivities from Table 1 were obtained at higher temperatures and have been extrapolated to $550 \mathrm{~K}$ for this work. However, there have been instances where hydrogenic permeation of $\mathrm{SiC}$ has approached the levels measured for $\mathrm{W}$ [13]. While the crystallinity and other properties of the SiC layers were not characterized in this study, the sensitivity of diffusivity and thermal conductivity to these properties does provide another variable for optimization of a $\mathrm{SiC}$ permeation barrier in future work. 
Most of the targets in this work used $25 \mathrm{~mm}$ diameter disks were used as bulk W substrates (99.5\% pure). The substrate surface was mechanically polished to an average surface roughness of $\sim 11 \mathrm{~nm}$ as measured by atomic force microscopy. The tungsten substrates were then cleaned in a 10:1 buffered oxide etch to strip oxides and free residual particles from the mechanical polishing. On these polished substrates, a layer of $\mathrm{SiC}$ was deposited via $\mathrm{RF}$ magnetron sputtering with a power of $200 \mathrm{~W}$ RF in a $5.6 \mathrm{mTorr}$ environment supplied with and argon flow of $40 \mathrm{sccm}$. This resulted in a SiC layer growth rate of $\sim 4 \mathrm{~nm} /$ minute for a total layer thicknesses in the 500-2000 $\mathrm{nm}$ range. The top layer of $\mathrm{W}$ was deposited on the $\mathrm{SiC}$ layer via $\mathrm{RF}$ magnetron sputtering with a power of $400 \mathrm{~W} \mathrm{RF}$ in a 15 mTorr environment supplied with an argon flow of $97 \mathrm{sccm}$ resulting in a W layer deposition rate of $25 \mathrm{~nm} /$ minute for a total layer thickness in the 1500-2500 nm range in this study. Deposition rates were determined by post-growth cleaving and SEM imaging.

A Si substrate was used for a single target for thermal testing purposes. On this target a W-SiC-W multi-layer construct was deposited on top of the Si substrate using the same RF magnetron sputtering settings as mentioned above. Initially a 4 $\mu \mathrm{m}$ thick layer of $\mathrm{W}$ was deposited on the Si substrate, followed by a $1 \mu \mathrm{m}$ layer of $\mathrm{SiC}$, and finally a $2 \mu \mathrm{m}$ top layer of $\mathrm{W}$.

\subsection{Thermal testing}

The targets were thermally cycled to test for cracking delamination of the deposited layers. The prepared targets were placed in an All-Win 610 rapid thermal anneal (RTA) system for thermal cycling. In an atmospheric pressure nitrogen 
environment, targets were brought from room temperature to $1023 \mathrm{~K}$ at a rate of $75 \mathrm{~K} / \mathrm{min}$, and then held at that temperature for 60 seconds. Then, over the following 60 second period, the sample was cooled to $673 \mathrm{~K}$. After this point the RTA was powered off and the sample was allowed to cool for 5 minutes (back to room temperature). This entire process was performed three times for each sample. After the thermal cycling the targets were inspected with focused ion beam (FIB) cross-sectioning and SEM imaging for any indications of layer delamination or cracking. It is also worthwhile to note that $\mathrm{W}$ and $\mathrm{SiC}$ both have low swelling characteristics under neutron irradiation, which can be important in a neutron-rich fusion reactor environment as mismatched swelling characteristics could also lead to mechanical breakdown or fracturing of the deposited layers under prolonged neutron bombardment. This was not confirmed or investigated in this work though.

\subsection{Diffusion testing}

To confirm that the SiC layer inhibits diffusion and thus permeation through the material, a target was exposed to a magnetized deuterium plasma from a RF helicon plasma source. The RF power was $800 \mathrm{~W}$ at a pressure of $1.6 \mathrm{~Pa}$ in a $500 \mathrm{G}$ magnetic field. This resulted in an incident ion plasma flux of $1.5 \times 10^{22} \mathrm{D} / \mathrm{m}^{2} \mathrm{~s}$. The target was exposed for 1800 seconds. Target surface temperature was elevated to $550 \pm 20 \mathrm{~K}$, as measured by pyrometry, to activate diffusion through the $\mathrm{W}$ layers. A bulk W target was exposed to near-identical conditions for comparison. The $550 \mathrm{~K}$ exposure temperature is lower than the expected wall temperature of future reactors. However it was selected for this work as this is a sufficiently high temperature that implanted D can easily diffuse through the first $\sim 5$ um of bulk tungsten while low 
enough that a significant amount of implanted D remains trapped in the target for post-exposure measurements and depth profiling. Thus it is deemed appropriate to test the efficacy of a SiC diffusion barrier.

After the plasma exposure, ex-situ $\mathrm{D}\left({ }^{3} \mathrm{He}, \mathrm{p}\right)^{4} \mathrm{He}$ nuclear reaction analysis was used to depth profile the implanted deuterium in both targets. The multiple energy technique [14] was used to improve the depth profile with ${ }^{3} \mathrm{He}$ ion beam energies of 800,1600 , and $2700 \mathrm{keV}$ selected such that the reaction cross section resonance occurs in the top W layer, the SiC layer and the bulk W substrate respectively. The ion beam was normal to the target with a scattering angle of $150^{\circ}$. An $800 \mu \mathrm{m}$ aluminum foil was placed in front of the detector to slow the high-energy protons from the nuclear reaction such that they could be fully stopped in the detector. Collected charge from the ion beam on the target was integrated to ensure identical beam fluence for each measurement. Once the data is acquired, simNRA $[15,16]$ is used to fit the data and translate it into depth profiles.

\section{Results and Discussion}

\subsection{Results of thermal testing}

This entire thermal process was performed three times for each sample. Two samples with stacks of $\mathrm{W} / \mathrm{SiC} / \mathrm{W}$ were examined - one created on a silicon substrate, and another on a bulk W substrate (see section 2.1). The silicon sample was cleaved and examined for long range damage (Fig $1 \mathrm{~A}$ ). The tungsten sample was placed in an FEI 835 focused ion beam (FIB) system and several 10x20 $\mu \mathrm{m}$ regions were milled out using gallium ions; a typical 'trench' is shown in Fig 1B. Concurrent with visual inspections, no delamination or cracking was observed 
between the tungsten and silicon carbide layers in either sample. The next step in testing is the resilience of these samples to 10-100's of thermal cycles, which the PFC may experience in a pulsed or transient fusion reactor environment. This test, along with thermal cycling after plasma loading, are planned for future work.

\subsection{Results of diffusion tests}

The deuterium depth profiles from Nuclear Reaction Analysis allow us to see the diffusion behaviour of the implanted deuterium ions and the impact of the $\mathrm{SiC}$ layer on this behaviour. Acquiring data at three different ion beam energies gives greater confidence in the depth profiles and the most drastic difference between the W-SiC target and the pure $\mathrm{W}$ target can be seen for the $2700 \mathrm{keV}$ beam energy (Fig. 2) since this beam energy is most sensitive to the deuterium beyond the depth of the $\mathrm{SiC}$ layer starting at $\sim 1300 \mathrm{~nm}$. The data indicates that there is no D beyond the SiC layer, confirming its effectiveness as a diffusion barrier for hydrogenic species. The resulting D depth profiles (Fig. 3) obtained through self-consistent simNRA fits of the experimental data (Fig. 2) shows the deuterium trapping in the targets after plasma exposure.

Given the large differences in diffusion rates and the sudden transition of materials, one might expect implanted deuterium to build up at the transition from $\mathrm{W}$ to SiC. The transition of materials may provide trap sites for the deuterium due to misalignments or grain boundaries in the transition from $\mathrm{W}$ to SiC lattice structures. An accumulation of $\mathrm{D}$ at this interface could lead to the formation of voids in a process similar to the formation of blisters on W surface exposed to hydrogenic plasmas [17]. Extensive void formation can play a role in delamination concerns. 
However, despite a small but measurable amount of D diffusing into the SiC layer, there is no build up of $\mathrm{D}$ at the transition from $\mathrm{W}$ to SiC. FIB cross-sectioning and SEM imaging after plasma exposure also revealed no bubble/void formation or layer delamination. With the lack of build-up at the $\mathrm{W}$ to SiC interface, the overall $\mathrm{D}$ retention of the $\mathrm{W}$-SiC target is significantly lower than the bulk $\mathrm{W}$ target indicating that a larger fraction of the implanted D was released back out the surface for the WSiC target.

\section{Conclusions}

A SiC permeation barrier laid down with RF magnetron sputtering in a tungsten plasma-facing component has been found to be effective at greatly reducing D diffusing through the bulk of the material and stable enough to survive thermal cycling without delamination or mechanical breakdown. The key characteristics for $\mathrm{SiC}$ are its similar thermal conductivity and thermal expansion but much lower hydrogenic diffusivity than W. In this scenario the diffusion layer is too close to the surface to be practical for a reactor application due to sputtering and erosion concerns. Future work is aimed towards achieving greater control and optimization of the SiC layer crystallinity and morphology, more stringent thermal testing, and validating fabrication techniques such that the diffusion barrier can be at much greater depths $(\sim 1 \mathrm{~mm})$ such that the diffusion barrier can survive for extended periods in a reactor environment.

\section{Acknowledgements}

This work was supported by United States Department of Energy (DOE) grant DESC0009685 and the US DOE STTR program. 


\section{References}

[1] T. Chikada, A. Suzuki, Z. Yao, D. Levchuk, H. Maier, T. Terai, T. Muroga, Fusion Eng. Des. 84 (2009) 590.

[2] T. Chikada, A. Suzuki, T. Terai, Fusion Eng. Des. 86 (2011) 2192.

[3] Y. Hatano, K. Zhang, K. Hashizume, Physica Scripta T145 (2011) 014044.

[4] L.S. Sigl, J. Eur. Ceram. Soc. 23 (2003) 1115.

[5] G.A. Slack, J. Appl. Phys. 35 (1964) 3460.

[6] D. Liu, B. Lin, Ceram. Int. 22 (1996) 407.

[7] P. Hidnert, W.T. Sweeney, dx.doi.org/10.6028/nbsscipaper.203

[8] Goldberg Yu., Levinshtein M.E., Rumyantsev S.L., Properties of Advanced Semiconductor Materials GaN, AlN, SiC, BN, SiC, SiGe, John Wiley \& Sons, Inc., New York, 2001, 93-148.

[9] R. Frauenfelder, J. Vac. Sci. Technol. 6 (1969) 388.

[10] R.A. Causey, W.R. Wampler, J.R. Retelle, J.L. Kaae, J. Nucl. Mater. 203 (1993) 196.

[11] R.A. Causey, J.D. Fowler, C. Ravanbakht, T.S. Elleman, K. Verghese, J. Am. Ceram. Soci. 61 (1978) 221.

[12] G.A. Esteban, A. Perujo, F. Legarda, L.A. Sedano, B. Riccardi, J. Nucl. Mater. 307311 (2002) 1430.

[13] S. Sinharoy, W.J. Lange, J. Vac. Sci. Technol. A 2 (1984) 636.

[14] C.J. Altstetter, R. Behrisch, J. Bøttiger, F. Pohl, B.M.U. Scherzer, Nucl. Instr. and Meth. 149 (1978), 59. 
[15] M. Mayer, Technical Report IPP 9/113, Max-Planck-Institut für Plasmaphysik, Garching, Germany, 1997.

[16] M. Mayer, Proceedings of the 15th International Conference on the Application of Accelerators in Research and Industry, American Institute of Physics Conference Proceedings 475 (1999) 541.

[17] Y. Ueda, M. Fukumoto, J. Yoshida, Y. Ohtsuka, R. Akiyoshi, H. Iwakiri, N. Yoshida, J. Nucl. Mater. 386-388 (2009) 725. 
Tables and captions:

\begin{tabular}{|l|l|l|}
\hline & Tungsten & Silicon Carbide \\
\hline $\begin{array}{l}\text { Thermal conductivity } \\
\text { at } 300 \mathrm{~K}\end{array}$ & $174 \mathrm{~W} / \mathrm{m} \cdot \mathrm{K}$ & $72-114 \mathrm{~W} / \mathrm{m} \cdot \mathrm{K}[4-6]$ \\
\hline $\begin{array}{l}\text { Coefficient of thermal } \\
\text { expansion at } 300 \mathrm{~K}\end{array}$ & $4.3 \times 10^{-6} \mathrm{~K}^{-1}[7]$ & $3.8 \times 10^{-6} \mathrm{~K}^{-1}[8]$ \\
\hline $\begin{array}{l}\text { Hydrogenic diffusivity } \\
\text { at } 550 \mathrm{~K}\end{array}$ & $1.1 \times 10^{-10} \mathrm{~m}^{2} / \mathrm{s}[9]$ & $9.7 \times 10^{-48}-6.6 \times 10^{-24} \mathrm{~m}^{2} / \mathrm{s}[10-12]$ \\
\hline
\end{tabular}

Table 1: Comparison of thermal properties and hydrogenic diffusivity between bulk tungsten and silicon carbide. 
Figures and captions:

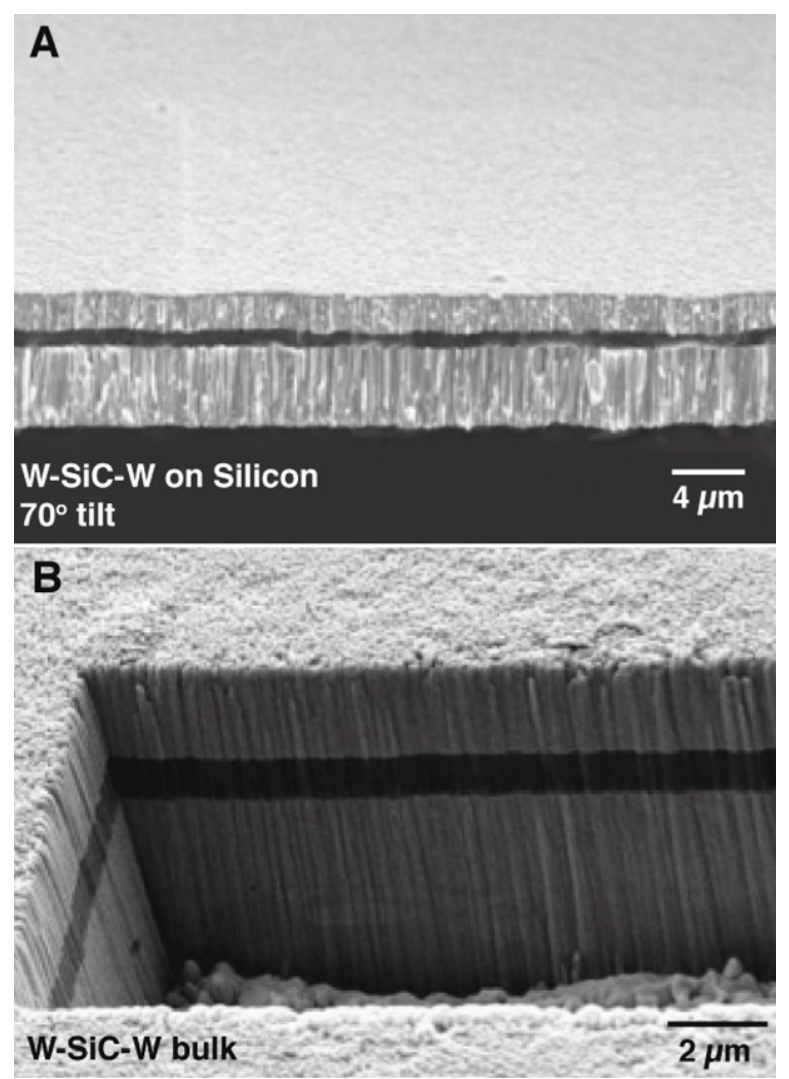

Figure 1: Cross-section imaging of W-SiC-W interfaces on a A) Si substrate and B) a bulk W substrate after thermal cycling treatments. No damage or delamination is observed. 


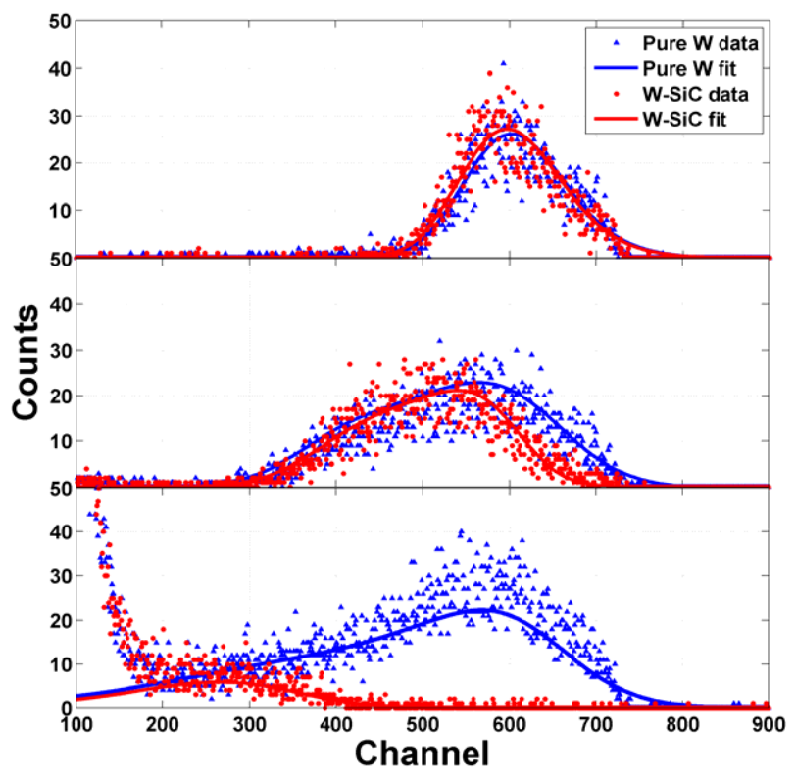

Figure 1: The NRA spectra at ion beam energies of 800,1600 , and $2700 \mathrm{keV}$ for pure $\mathrm{W}$ target and a W target with SiC permeation layer at a depth of $1300 \mathrm{~nm}$ from the surface. The spectra fits are generated with the simNRA code $[9,10]$.

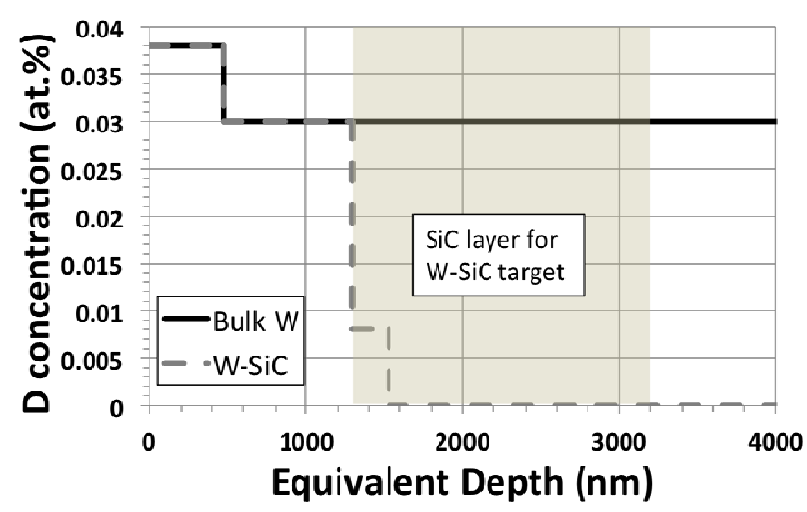

Figure 2: Deuterium concentrations as a function of depth for bulk $\mathrm{W}$ and $\mathrm{W}$-SiC targets. The shaded region represents the $\mathrm{SiC}$ layer for the $\mathrm{W}$-SiC target. Equivalent depth is calculated from areal densities measured with ion beam analysis and assuming bulk solid densities for $\mathrm{W}$ and $\mathrm{SiC}$. 